\title{
Vínculos de adolescentes en medida de restablecimiento de derechos*
}

\section{Bonding in adolescents undergoing rights restoration processes}

\author{
Teresita Bernal Romero** \\ Universidad Santo Tomás, Colombia \\ Miguel Melendro Estefanía \\ UNED, España \\ Recibido: 20 de enero de 2014 \\ Revisado: 3 de marzo de 2014 \\ Aceptado: 1 de abril de 2014
}

\section{Resumen}

El objetivo de este artículo es presentar los resultados de la investigación Vínculos de adolescentes en medida de restablecimiento de derechos. La investigación tuvo como objetivo describir y evaluar los vínculos de adolescentes que han sido declaradas en adopción y que se encuentran en una Institución de protección. Se utilizó una investigación de tipo biográfico con relatos de vida, entrevistas y mapas de vínculos, con cuatro adolescentes seleccionadas desde un muestreo teórico. Para el procesamiento de la información, se trabajó con técnicas de análisis de contenido. Se concluyó que las adolescentes han construido diferentes vínculos con la familia de origen dependiendo de su historia y de los significados y las cualidades de los vínculos. Los educadores de hogar y los padrinos se constituyen en figuras subsidiarias de vinculación desde los cuidados cotidianos que ofrecen a las adolescentes.

Palabras clave: adolescentes, vínculos, servicios de protección.

* Artículo de investigación.

** Correspondencia: Teresita Bernal Romero, Universidad Santo Tomás. Correo electrónico: HYPERLINK “mailto:teresitabernal@usantotomas.edu. co" teresitabernal@usantotomas.edu.co. Facultad de Psicología, Bogotá, Colombia

Miguel Melendro Estefanía. UNED. Correo electrónico: HYPERLINK “mailto:mmelendro@edu.uned.es” mmelendro@edu.uned.es Facultad de Educación. Dpto. Teoría de la Educación y Pedagogía Social, Madrid, España 


\section{Abstract}

The aim of this paper is to present the results of a study called Bonding of adolescents under rights restoration processes. The objective was to describe and evaluate the bonds of teenagers who have been placed for adoption and are now at a protection institution. The investigation was biographical in nature with life stories, interviews and bonding relationships maps, with four adolescents selected from a theoretical sampling. Data were processed through Content Analysis. The conclusion was that the adolescents had built different bonds with the original family depending on their stories and the meanings and qualities of the bonding. Household educators and godfathers became subsidiary bonding figures from the daily care they provide to the adolescents.

Keywords: Bonding relationships, protection services, adolescents

\section{Presentación}

El desarrollo emocional es fundamental en la vida de los niños, niñas y adolescentes (Armus, Duhalde, Oliver, Woscoboinik, 2012) y en él, son muy importantes las relaciones y los vínculos con las familias biológicas (García-Calvo, Guerrero y García, 2000; Moreno-Manso, García-Baamonde, Sánchez, Guerrero-Barona, y Blázquez-Alonso, 2010; Henao y García, 2012). Una parte importante de los estudios, alrededor de este tema, han señalado una serie de cuestionamientos sobre la figura que genera vínculo: ¿Son una o varias personas aquellas con las cuales los sujetos pueden establecer vínculos significativos? ¿Solo son posibles vínculos significativos con la figura principal de apego? ¿Cómo se movilizan los vínculos cuando las figuras primarias y subsidiarias cambian constantemente? Estas preguntas son además fundamentales en personas que han vivido diferentes rupturas con sus figuras primarias, como es el caso de los adolescentes en protección (Melendro 1998; Melendro y Cruz, 2013; Melendro, 2013)

El presente artículo muestra los resultados de la investigación titulada Vínculos de adolescentes en medida de restablecimiento de derechos. El estudio se centró en adolescentes en medida de restablecimiento de derechos, que viven en una Institución de protección de la ciudad de Bogotá (Colombia). Estas adolescentes no fueron reintegradas a sus familias biológicas porque las familias no tenían las condiciones para garantizar sus derechos, y por ello fueron en su momento declaradas en medida de adoptabilidad. En su desarrollo, estas adolescentes no fueron ni van a ser acompañadas por una figura principal, como una madre o un padre, sino que por el contrario cambian constantemente de figuras de referencia: educadores de hogar, equipo psicosocial, padrinos, entre otros. Así, el interés de la investigación se centra en la descripción de los vínculos que han construido las adolescentes con diferentes figuras centrales y subsidiarias.

\section{Marco de referencia}

En el estudio de los vínculos, Bowlby (1997) privilegia una sola figura permanente y plantea que en esta relación se dan varios tipos de apego, los cuales van a tener repercusión en la vida adulta del niño. Oliva (2004), por su parte, ha cuestionado algunos planteamientos de las teorías tradicionales frente a este tema y aporta una visión crítica hacia lo que él denomina el monotropismo en la teoría del vínculo, es decir, hacia la necesidad de centrar el proceso de desarrollo y vincularlo en un único sujeto. Ante esto, Oliva (2004) propone que pueda contemplarse el desarrollo vincular a partir de diferentes figuras alternas. Por otra parte, Amar, Kotliarenko y Abello (2003) también consideran que es posible establecer vínculos con otras personas diferentes a la familia. Castelblanco, Moreno, Moreno, Sánchez, Garzón y Duque (2012) por su parte, encuentran que los adolescentes protegidos construyen vínculos con otras personas distintas a su familia de origen. 
Más allá de estas conceptualizaciones sobre el vínculo, Dabas y Najmanovich (1995) plantean la noción de vínculo como una emergencia de los procesos de auto-organización. Estos autores plantean la importancia del reconocimiento de la construcción del sujeto en los intercambios sociales; es decir, los sujetos se configuran en múltiples vínculos sociales de afecto, de lenguaje, de comportamientos, que el sujeto va autoorganizando. En este sentido, se puede entender el vínculo como el constante intercambio de interacción con los otros a nivel afectivo, comunicacional y comportamental, para organizarse en su contexto.

Otros autores asumen una postura eco-eto-antropológica cuando abordan este tema; así, en palabras de Hernández y Bravo (2004), el ser humano construye ecosistemas y estos a su vez lo configuran a él. Desde este punto de partida, Hernández (2010) plantea los siguientes presupuestos para el estudio del vínculo:
- La noción de vinculo "tiene un valor de supervivencia" (p. 56).

- Es un concepto relacional entre dos dimensiones de lo humano, que a veces se consideran como opuestas.

- Asumir la categoría del vínculo implica reconocer y relacionar las dimensiones filogenética, ontogenética, epigenética y cultural.

- Los vínculos se diferencian por el contexto donde se generan, por las distintas etapas del ciclo vital, por las condiciones históricas, socioculturales y por los significados.

- Los procesos de vinculación favorecen la construcción de autonomía.

Además, Hernández (2010) explica que los vínculos tienen cualidades que presentan variaciones entre extremos. En estos extremos es importante mencionar que no hay puntos ideales, sino acuerdo al contexto, la historia y el momento del ciclo vital, como se ha mencionado anteriormente. La autora las sintetiza en la siguiente tabla:

Tabla 1.

Cualidades de la vinculación (Hernández, 2010, p. 282).

\begin{tabular}{|c|c|c|}
\hline Cualidades & Vinculación & \\
\hline $\begin{array}{l}\text { Función de supervivencia: en qué medida el vínculo es } \\
\text { condición para la supervivencia. }\end{array}$ & Contingente & $\longleftrightarrow$ Vital \\
\hline $\begin{array}{l}\text { Función evolutiva: cómo favorece el desarrollo humano } \\
\text { de los sujetos. }\end{array}$ & $\begin{array}{l}\text { Limitante, } \\
\text { anquilosante }\end{array}$ & $\longleftrightarrow$ Movilizante \\
\hline $\begin{array}{l}\text { Función creativa: cómo favorece la emergencia de nove- } \\
\text { dades adaptativas. }\end{array}$ & Destructiva & $\longleftrightarrow$ Generativa \\
\hline $\begin{array}{l}\text { Libertad: en qué medida los sujetos eligen y/o aceptan } \\
\text { estar en esa relación. }\end{array}$ & Forzada & $\longleftrightarrow$ Elegida, aceptada \\
\hline $\begin{array}{l}\text { Propósito consciente: si el sujeto está en la relación deli- } \\
\text { beradamente o de hecho. }\end{array}$ & Contractual & $\longleftrightarrow$ De hecho \\
\hline Consistencia: organización y estabilidad de la vinculación. & Incierta, ambigua & $\longleftrightarrow$ Clara \\
\hline Fuerza del vínculo: intensidad y resistencia del vínculo. & Frágil & $\longleftrightarrow$ Fuerte \\
\hline Permanencia: duración y continuidad del vínculo. & $\begin{array}{l}\text { Fugaz, } \\
\text { intermitente }\end{array}$ & $\longleftrightarrow$ Permanente \\
\hline $\begin{array}{l}\text { Presencialidad: de qué forma de presencia de los sujetos } \\
\text { depende el vínculo. }\end{array}$ & Virtual & $\longleftrightarrow$ Presencial \\
\hline Ritualización: grado de ritualización del vínculo. & Desvirtualizada & $\longleftrightarrow \begin{array}{l}\text { Ritualizada } \\
\text { rígidamente }\end{array}$ \\
\hline
\end{tabular}


En investigaciones sobre vínculos de niños, niñas y adolescentes en situación de protección, Amar y Berdugo (2006) encuentran que la madre es una figura central de apego en el niño, y es la encargada de proveer todo el afecto. Sin embargo, sus investigaciones demostraron que no hay necesidad de una figura central "madre" que supla las necesidades del niño, sino que existen figuras subsidiarias, como hermanos mayores y otros miembros de la familia extensa. González y Méndez (2002) hallan que en los niños institucionalizados los cuidadores son muy importantes en su desarrollo. Por su parte, Arango (2003) plantea que el fortalecimiento de vínculos afectivos permite la relación y favorece la convivencia con los otros.

Finalmente, el retomar los planteamientos de estos autores implica reconocer que la construcción de los vínculos en los seres humanos es un proceso continuo que se da en múltiples sistemas, contextos y relaciones. Invita también a trascender el determinismo de los vínculos de la infancia y conlleva a comprender que los vínculos se resignifican en diferentes momentos, que hacen parte de redes más amplias y complejas de relaciones y que desde allí se construyen y se actualizan sus sentidos; elementos fundamentales en investigaciones que se centran en niños, niñas y adolescentes que han cambiado de figuras de vinculación.

\section{Diseño metodológico}

La investigación se realizó desde los principios de la investigación tipo biográfico:
1. Los métodos biográficos parten de los discursos que los sujetos elaboran; en este caso de los discursos que las adolescentes han elaborado sobre los vínculos que han construido a lo largo de su vida.

2. Los discursos sobre la vida de las personas tiene un interés investigativo porque el tipo de situaciones que han vivido son importantes para comprender procesos sociales. En esta investigación a partir de los discursos de las adolescentes se comprenden procesos alrededor de adolescentes que viven en instituciones y sin familias.

3. Los métodos biográficos enfatizan en singularidades y simultáneamente brindan elementos para comprender aspectos similares en diferentes biografías. En este sentido, se realizó primero un análisis de cada discurso de las participantes y luego se procedió a revisar diferencias, similitudes, regularidades entre los distintos discursos.

4. No es fundamental la representatividad, pues su objetivo no es la generalización, sino la pertinencia. Desde este planteamiento se trabajaron cuatro relatos de vida de adolescentes institucionalizadas.

A partir de estos referentes en la investigación participaron cuatro adolescentes y el equipo psicosocial que las atiende. Fueron seleccionadas desde un muestreo teórico teniendo en cuenta diferentes criterios: las adolescentes debían estar en medida de restablecimiento de derechos y habían sido declaradas en situación de adoptabilidad, de-

Tabla 2.

Descripción de las adolescentes.

\begin{tabular}{clccc}
\hline Adolescente & Motivo de ingreso a protección & Edad de ingreso a protección & $\begin{array}{c}\text { Edad en la } \\
\text { investigación }\end{array}$ & Escolaridad \\
\hline 1 & $\begin{array}{l}\text { Muerte de la madre. No hay } \\
\text { quien asuma los cuidados de } \\
\text { los niños }\end{array}$ & $\begin{array}{c}\text { Reintegrada a la familia } \\
\text { a los } 5 \text { años }\end{array}$ & 17 & $\begin{array}{c}\text { Noveno } \\
\text { grado }\end{array}$ \\
2 & $\begin{array}{l}\text { Conflictos conyugales. } \\
\text { Intento de abuso sexual }\end{array}$ & 12 años & 16 & $\begin{array}{c}\text { Octavo } \\
\text { grado }\end{array}$ \\
3 & $\begin{array}{l}\text { Abandono de la progenitora. } \\
\text { Progenitor que asume custodia } \\
\text { ingiere licor }\end{array}$ & 14 años & 17 & $\begin{array}{c}\text { Noveno } \\
\text { grado }\end{array}$ \\
Negligencia. & Abuso sexual & 9 años & 17 & $\begin{array}{c}\text { Décimo } \\
\text { Grado }\end{array}$ \\
\hline
\end{tabular}


bían tener más de 16 años y vivir en una Institución de protección, debían tener diferentes motivos de ingreso al sistema para que se pudiera recuperar diferentes historias, y debían querer participar de manera voluntaria en la investigación.

El equipo psicosocial estuvo constituido por un psicólogo y una trabajadora social que han participado en el seguimiento de las adolescentes.

Se usaron en la investigación varios instrumentos que fueron piloteados con anterioridad:

Relatos de vida: este instrumento tenía como objetivo recuperar la historia de los vínculos de las adolescentes. Se generó una estructura que obedeció a tres momentos: antes de la medida de restablecimiento de derechos, la toma de la medida de restablecimiento y la actualidad. En promedio, se realizaron cuatro sesiones de relato de vida, de dos horas cada una, por cada participante.

Mapa de vínculos. este instrumento es de orden gráfico. En una hoja en blanco, la adolescente representaba en esta a sí misma y a figuras significativas en su vida, a través de círculos. Luego se le solicitaba que diagramara las relaciones significativas. Este instrumento permitió triangular la información dada por el relato de vida. Para elaborar el mapa se usó una sesión de una hora con cada adolescente.

Entrevista: esta se desarrolló con el equipo psicosocial que ha atendido el proceso de las adolescentes y la educadora del hogar. El formato fue analizado por expertos y respondía a categorías como: historia de la adolescente, vínculos de la adolescente en esta historia, características de los vínculos con otros y con el profesional. Esta entrevista duró una hora con cada profesional.

Análisis de contenido: a través de categorías inductivas y deductivas sobre las cualidades de los vínculos propuestas por Hernández (2010), se organizó y analizó la información. La categorización fue realizada por diferentes observadores para lograr su validez.

La investigación se desarrolló a través de diferentes etapas:
Etapa 1. Acercamiento a las fuentes: se coordinó con la Institución la ejecución del proyecto de investigación y se seleccionaron casos. Se verificó la disponibilidad de participación de los sujetos en la investigación. A cada una de las adolescentes, en sesión presencial, se le explicaron los objetivos de la investigación y el proceso de investigación. Se les aclaró que la participación en la investigación era voluntaria, que la información sería transcrita y se usaría en publicaciones bajo anonimato y que podrían retirarse de la investigación en el momento que lo desearan; lo cual no tendría ninguna implicación en la Institución.

Etapa 2. Aplicación y análisis de instrumentos: a partir de la aceptación de las adolescentes, se inició con los relatos de vida. El sitio para reconstruir el relato fue escogido por las adolescentes dentro de la Institución; estos debían cumplir con ciertas características para aseguran la confidencialidad. Para ello se usaron cuatro sesiones de promedio. El relato fue reconstruido según el formato usando una estructura de orden cronológico. Las transcripciones fueron revisadas con las adolescentes y ellas agregaron alguna información, en un proceso de devolución sistemática. Después de la transcripción se procedió a dos lecturas para la comprensión de la estructura general de estos, luego se organizaron estos en una matriz de doble entrada. Cada relato a partir de este análisis de categorías es reconstruido y reorganizado; lo cual se presentó nuevamente a las adolescentes para que ellas realizaran ajustes; luego se procedió a realizar una lectura profunda de cada relato, desde las categorías, comprendiendo su sentido en la narrativa de cada una.

En otra sesión se realizaron los mapas de vínculos. Finalmente, se les solicitó a las adolescentes que narraran el por qué habían representado ese tipo de relación. Su análisis también se realizó a través de las categorías de cualidades de los vínculos, que se evidenciaron en el uso de las convenciones de las relaciones y en las justificaciones que las adolescentes generaban alrededor de la persona significativa.

La entrevista al equipo psicosocial se realizó con los dos integrantes en una misma sesión, en una 
oficina de la Institución. Para la entrevista se usó un formato de preguntas sobre cada uno de los momentos definidos para el relato de vida por cada una de las adolescentes. Finalmente, se realizaron preguntas aclaratorias sobre el cómo, cuándo, con quién. La información también fue grabada con consentimiento de los integrantes del equipo. Se procedió a transcribir la entrevista y a realizar lecturas sobre la estructura de esta. Después de todo esto se organizaron en una matriz de doble entrada: cualidades de los vínculos y momentos del relato por cada una de las adolescentes.

Etapa 3. Validación de los resultados: se procedió a realizar la validación de la información a través de triangulación de las fuentes y de estrategias investigativas con una matriz de triple entrada: 1) categorías, 2) fuentes de la investigación y 3) momentos de la historia de cada una de las adolescentes. En esta matriz se evidenciaron datos contradictorios y complementarios, se pudo establecer la particularidad de cada uno de los casos y elementos globales. Finalmente, se llevó a cabo la devolución sistemática a las adolescentes y al equipo psicosocial.

\section{Resultados}

A partir del estudio se evidenció que las adolescentes han establecido vínculos con algún tipo de figura. Algunas de estas figuras cumplen cualidades de figuras subsidiarias que son significativas y han aportado al desarrollo de estas adolescentes, por lo que se podrían denominar figuras significativas en los vínculos; con otras se han presentado dificultades y a veces han logrado resignificar estos vínculos, sin que por ello se pueda afirmar que el vínculo fuera significativo o no en otro momento de la historia de las adolescentes. A continuación se presentan los resultados con: familia de origen, hogares sustitutos, equipo psicosocial, y padrinos, teniendo en cuenta el siguiente mapa (Figura 1).

\section{Vínculos con la familia de origen}

En los vínculos con la figura materna se resal$\tan$ las relaciones que se tuvieron y otras que se mantienen a pesar de la distancia y del rompimiento del vínculo legal con las familias. Al respecto se encuentran dos tendencias: la primera a mantener vínculos, aunque no exista en este momento ningún tipo de contacto, y la segunda a la resignificación de los vínculos con las figuras del pasado. Frente a la primera tendencia, en el caso 3, la adolescente aún mantiene una relación vincular que ella considera significativa con la figura materna. La madre los abandonó cuando la adolescente tenía 11 años y constituyó una nueva familia; los hijos quedaron al cuidado del padre. Sin embargo, la adolescente considera que si hubiera buscado a su madre no estaría en el sistema de protección. Por otra parte, ella continúa recibiendo información sobre la mamá, en este caso a través de la hermana, lo que va alimentando la relación vincular. Parece que el tener información sobre la mamá hace mantener el vínculo como significativo para esta adolescente, dificultando inclusive la construcción de un proyecto de vida con las condiciones actuales que tiene.

Figura 1. Categorías de los resultados.

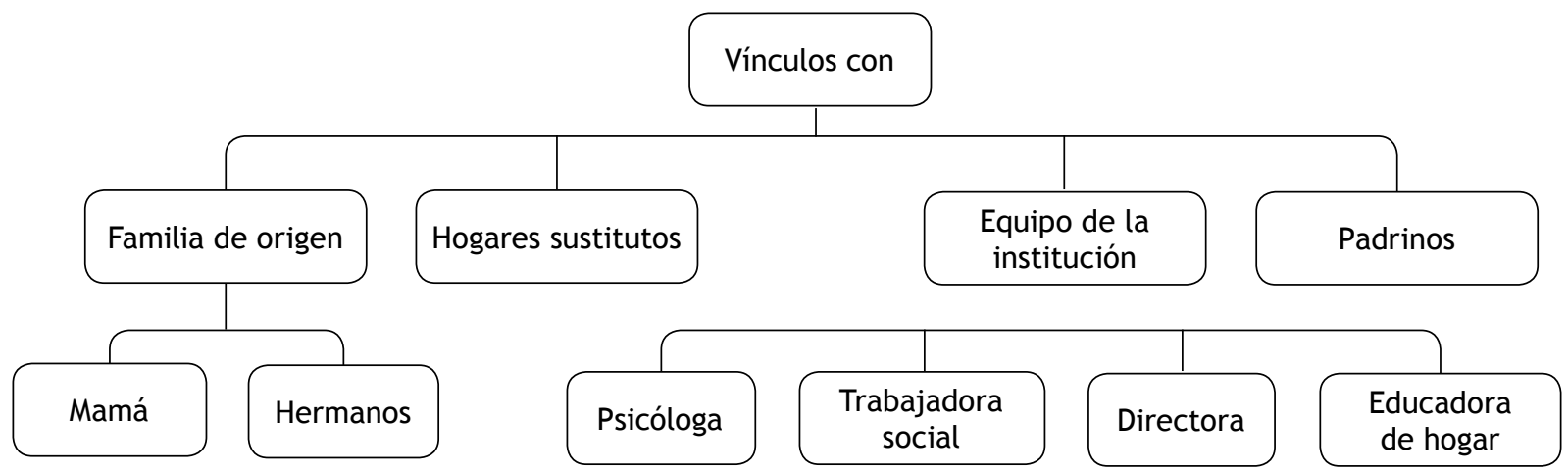


En esta misma tendencia está la adolescente 1, que recuerda y mantiene presente la relación vincular con la madre, relación que reconstruye luego con la madre sustituta:

Esa fue la noche que ella había salido, nosotras no sabíamos, entonces en el Bienestar nos anunciaron y nos dijeron que mi mamá había fallecido, nos reunieron y pues a mí fue la primera que le contaron y después a mi hermana y lloramos (adolescente 1).

En estos dos casos la convivencia con la figura materna se vio afectada por algo externo: en la adolescente 1 la madre muere y en el número 3 la madre se va del lado de sus hijos (la adolescente no lo significa como un abandono). En este sentido, pareciera que en estos casos el vínculo se mantiene como significativo, cuando la relación con la madre es afectada por algo que ellas (las adolescentes) consideran externo a la figura misma; casi como que la madre se constituye en víctima de las condiciones que impiden que esté con sus hijos en la actualidad.

Frente a las cualidades de los vínculos, en estas dos adolescentes no son muy evidentes por la ausencia de las figuras maternas durante un período largo; en estos procesos vinculares no se dio permanencia, ni presencialidad. $Y$ en la adolescente 3 la cualidad de supervivencia fue contingente, pues la madre se marchó; aunque no se puede asegurar cómo era la relación antes de la ausencia.

En la segunda tendencia, se ubican las otras dos adolescentes, significando el vínculo materno como algo que fue importante pero que ya no está. Estas adolescentes compartieron con sus figuras maternas el día a día:

... Cuando estábamos en instituciones, el primer año nos dio muy duro, pero ya al estar lejos de ella (la mamá), estar sin ella, lo hace a uno como más duro, como que ya. Pero yo a mi mamá no le guardo rencor, pero tampoco me hace falta (adolescente 2).

En este caso la relación vincular se caracterizó por las cualidades de presencialidad, ritualización y permanencia; sin embargo, la función de super- vivencia y evolutiva no eran evidentes. La mamá permanecía todo el día con los hijos, pero estos no estaban escolarizados, además presentaban estados de desnutrición.

En la adolescente 4, se encuentra que la relación vincular no es significativa. En este caso, la participante experimentó en la convivencia con la madre varias situaciones ante las cuales esperaba protección; pero esta no se dio. Se presentaron también experiencias de maltrato y abuso por parte del padrastro, que afectaron la relación vincular en varias de sus cualidades: 1 ) en cuanto a la función de supervivencia, pues esta se constituye como contingente, 2) en la evolutiva, pues no favorece el desarrollo, constituyéndose como anquilosante, 3) en la consistencia, pues se constituye como ambiguo "soy tu madre, pero no te protejo", 4) y en cuanto a la fuerza del vínculo, puede considerarse frágil.

En realidad yo no me acuerdo de mi infancia, de juguetes, de haber estado triste, de haber estado enferma; me acuerdo más de mis hermanos... (adolescente 4).

En los casos 3 y 4, la relación con la madre es resignificada. En la actualidad no piensan en buscarla, esperan que esté bien, pero su proyecto de vida no gira alrededor de ella. Al parecer este proceso se relaciona con las cualidades de la vinculación que establecieron con la figura materna. En el caso número 4 la función de supervivencia fue contingente, quien proveía de alimentos y de cuidados en el hogar no era la madre. La función evolutiva es limitante en ambos casos, son adolescentes que estuvieron desescolarizadas. La cualidad de consistencia se veía incierta porque no era muy claro el rol de la madre. Esto se evidencia más claramente en el caso 4 que en el 2 . Otras cualidades del vínculo se hicieron presentes como la presencialidad y la permanencia.

Uno de los factores que relaciona el equipo psicosocial con la significación de la relación vincular parece estar relacionado con la edad de ingreso de los niños a protección y la etapa en que son declarados en situación de adoptabilidad:

Cuando aquí nos llega un niño de 4 años, y pasan 3 o 4 años, ya se vuelve como de la 
familia, ya sienten que ellos nacieron acá en la Institución. Cuando se reciben de 6 años o más, ellos ya empiezan a tener más recuerdos de su familia, empiezan a dimensionar algunas situaciones y empiezan a recordar (psicólogo).

Se puede evidenciar cómo antes de los 5 años parece que la relación con la figura materna, al no generarse "recuerdos" de ella o tal vez de la relación vincular, esta es fácilmente resignificada y se generan nuevas relaciones con mayor facilidad. Sin embargo, esto no se puede evidenciar en los casos que estamos analizando, porque la edad mínima de ingreso de ellas fue de 8 años y la adolescente que ingresó a los 8 años todavía tiene algún contacto con la figura materna. Aunque sí se identifica en esta investigación la importancia de la dimensión temporal; pues se observa cómo algunos recuerdos facilitan o no la resignificación. Por ejemplo, en el caso 3 no se significa a la madre como la que abandona e inclusive de alguna forma todavía se le está esperando, y esta historia contrasta fuertemente con la adolescente 4 , en la que ella siente que a lo largo de su historia, aunque viviera con su madre, esta no estaba presente para cuidarla.

Por otra parte, los padres son poco mencionados en los relatos, aunque estos hayan asumido el cuidado de los hijos, como en el caso número 3. La adolescente 2 convivió con el padre y la madre al mismo tiempo, pero este es poco mencionado. $Y$ en el caso de las adolescentes 1 y 4, el tiempo de convivencia fue muy poco, luego compartieron con otras figuras como los padrastros, ante los cuales no se evidencia ningún tipo de vínculo significativo; antes bien, en la adolescente 4, se evidencia rechazo, Seguramente en relación a los abusos sexuales mencionados en su biografía.

En tres de los casos las adolescentes son las hermanas mayores. En el caso número 4, la adolescente no es la mayor, pero su hermano mayor se va de la casa en una edad muy temprana y ella asume su rol ante la partida. Como lo han demostrado otras investigaciones, las hermanas mayores asumen un rol parentalizado, ayudando en casa con labores y el cuidado de sus hermanos. Las adolescentes de los casos números 1 y 4 también asumen este rol.

¿Qué crees que pasaría con ella o qué harías tú si a tu hermana no la adoptaran?

Pues no sé qué pasaría. Pero si yo me voy la ayudaría mucho; hago todo lo posible para que tenga todo, como si yo fuera la mamá de ella (adolescente 1).

En el caso 1 el vínculo es fuerte y también continúa la cualidad evolutiva movilizante, en la medida en que la hermana está preocupada y desea gestionar elementos para el desarrollo de su hermana. Hay otras situaciones, como en el de la adolescente del caso número 4, que a pesar de la adopción de sus hermanos ella mantiene contacto con ellos e inclusive viaja a visitarlos en período de vacaciones.

En otros, como el caso número 2, no se vuelven a relacionar los hermanos que han sido adoptados. Cuando los grupos de hermanos son llevados a las instituciones las adolescentes tratan de mantener su rol parentalizado, intentando mantener la familia "unida".

Entonces nos separaron y a mí me dejaron sola en una Institución y a mis hermanos en otra, después nos reunieron a todos en un hogar sustituto y nos dijeron que posiblemente mis dos hermanitos se iban a adopción (adolescente 1).

A veces los niños y las niñas son enviados a instituciones diferentes por características particulares como discapacidad, trastornos, entre otros, otras veces ocupan en la misma Institución dormitorios o casas distintas, por ser de edad y género distinto, $y$ en algunas ocasiones son adoptados por padres diferentes. Si bien se intenta mantener a los hermanos unidos, es muy difícil que una familia adopte a 6 o 7 niños. Otro elemento que moviliza el rol parentalizado es resignificado por sus hermanos, pues la Institución asume este rol y además los niños y niñas empiezan a convivir con otros padres, que en algunos casos les resultan también gratificantes. La cualidad del proceso vincular de supervivencia es asumida por la Institución, al igual que las funciones evolutivas, de ritualización. 


\section{Vínculos en los hogares sustitutos}

En uno de los casos se hace referencia a los vínculos con hogares sustitutos. La adolescente manifiesta haber mantenido relaciones con una familia; es más, reconoce a la madre sustituta como parecida a la madre biológica:

Nos trataban muy bien, como si fuera nuestra familia propia, nos daban de todo. A mí, digamos, en el colegio siempre me felicitaban porque yo era la primera en todo... ellos se sentían orgullosos de mí y de mi hermana, porque éramos siempre las mejores... Nosotras les decíamos papá y mamá... (adolescente 1).

Estos vínculos se caracterizaban por el cuidado de los padres sustitutos en las actividades de todos los días, reconocimiento de lo que hacen las niñas en protección, compartir tiempo y actividades cotidianas juntos. La relación vincular todavía se mantiene y su sistema de significación está en relación al sentido de ser mamá. Por otra parte, el proceso vincular cumple con las cualidades de supervivencia vital, función evolutiva movilizante, vínculos fuertes, permanentes y presenciales.

\section{Equipo de la Institución}

Con el equipo psicosocial se establece una relación de ayuda. Acuden al psicólogo y al trabajador social en diferentes circunstancias, frente a dificultades específicas o por ser un requerimiento institucional:

Yo me sentía mal, pensaba que me quería morir por todo lo que me había pasado, y después la psicóloga empezó a ayudarme y me ayudó mucho y dijo que con llorar no sacaba nada, que fuera valiente (adolescente 1 ).

Uno recibe mucha ayuda psicológica... la psicóloga $C$, hablaba mucho conmigo, me daba muchos consejos (adolescente 2).

El equipo psicosocial plantea que las adolescentes no los consultan con frecuencia, sino cuando tienen una cita asignada o para resolver alguna contingencia específica. En las observaciones realizadas en la Institución, se evidencia como los niños de 7 a 10 años buscan más al equipo psicosocial. Sin embargo, en las narraciones con las adolescentes, se evidencia cómo el equipo psicosocial es fundamental para conversar y resolver dificultades relacionadas con su situación particular. Con ellos conversan sobre sus historias de maltrato, abuso, abandono, trámites legales, proyectos de vida en relación a su situación. Desde esta perspectiva, el equipo se constituye en un dispositivo de ayuda frente a su condición excepcional. Por otra parte, dos adolescentes plantean relaciones con la directora de la Institución: “¿Hay otra persona que sea muy importante para ti? -La directora de la Institución nos escucha y nos ayuda a solucionar cosas" (adolescente 2).

Esta directora forma parte del día a día de la Institución, inclusive comparte la hora de almuerzo y está pendiente de sus hábitos y comportamientos en el comedor. También se observa cómo conoce los nombres y las situaciones de niños y adolescentes. De alguna forma simboliza la Institución y los cuidados que se les brindan.

En estos casos la relación vincular está referida por figuras que se mantienen en la Institución, que pueden ayudar a solucionarles dificultades concretas en esta y que están pendientes de su situación. También resaltan el hecho de que estas personas les brindan algo concreto, como educación.

La educadora de hogar es una de las figuras con las cuales las adolescentes establecen vínculos significativos. La fortaleza de la relación se atribuye a que estas son las personas que las esperan en los dormitorios, están pendientes de ellas durante la noche. También plantean que se les puede comentar lo que ha sucedido en el día y hasta consultar problemas de la cotidianidad; algunas de las adolescentes las relacionan con figuras maternas. Además, de ellas reciben consejos y frases directas como: "hay que estudiar para ser alguien". En alguna forma, con estas educadoras se conversa sobre el día a día y con el equipo psicosocial sobre lo extraordinario. Esta diferencia se da por el carácter de la relación, su función, sus espacios físicos y simbólicos, por los tiempos que se comparten e inclusive por las mismas tareas que tiene que desarrollar cada figura. 
E: ¿Cuál es la persona a la que más acudes?

... A la educadora... me la llevo muy bien, le digo mami. Lo entiende a uno, es muy comprensiva. Ella hace el papel de mamá: nos corrige y es cariñosa (adolescente 4).

Las educadoras comparten con ellas fines de semana y noches; en ese tiempo se conversa sobre sus historias, lo que implica el reconocimiento de una relación sujeto-sujeto. De alguna manera, es un dispositivo de ayuda natural; es decir, dentro de la cotidianidad misma y no desde la excepcionalidad.

\section{Vínculos con los padrinos}

Los vínculos que se establecen con padrinos cambian en frecuencia de acercamientos y posibilidad de proveer económicamente recursos. En el caso 4 , se construyen las relaciones de apoyo y esto se hace evidente desde acciones concretas como comunicación constante, posibilidad de proveer recursos a la adolescente, de brindar otras formas de vida e inclusive la esperanza de la generación de un vínculo a través de la adopción.

En las adolescentes 2 y 3 , el apoyo de los padrinos se ha centrado más en la figura de la mujer (madrina) y la relación se constituye desde visitas esporádicas, la posibilidad de pasar tiempo con la madrina fuera de la Institución y la posibilidad de llamarla cuando se desea hablar con ella. En este caso, no se evidencian las cualidades del proceso de vinculación. Sin embargo, para la adolescente 2 , el encontrarse con la madrina es un momento muy importante, porque significa romper con la cotidianidad que implica el vivir institucionalizado.

\section{Discusión y conclusiones}

Para iniciar es importante retomar la idea de Cyrulnik (2008), sobre la gran diferencia de casos en los niños y niñas en situación de abandono; es así como los cuatro casos presentan elementos diferentes frente a los vínculos. Las diferencias están dadas por las historias, por las conformaciones familiares, por factores individuales de perso- nalidad, por los motivos de ingreso a protección, y por su proyecto de vida, entre otros. Sin embargo, las cuatro adolescentes han construido a lo largo de su vida distintos vínculos con distintas figuras, que han estado a cargo de su cuidado y de las cuales ellas reconocen aportes para su proyecto de vida. Entre ellas reconocen: las figuras maternas, los hermanos, el equipo psicosocial, las educadoras de hogar, los padrinos y los hogares sustitutos. Este hallazgos ratifica los resultados de Amar y Berdugo (2006), en los que se plantea cómo en la vida de los niños se establecen relaciones con diferentes figuras como se muestra a continuación.

Frente a los vínculos con las figuras maternas es importante mencionar la resignificación y la dimensión temporal. Se encontró que los vínculos de las adolescentes en situación de protección con sus figuras maternas en dos de los casos se mantuvieron y en los otros dos se movilizaron. En los casos que se mantuvieron el rompimiento fue significado como un alejamiento externo a la voluntad de la madre. En los últimos casos cambiaron de acuerdo a varios elementos: duración de la convivencia con la madre, protección de la madre ante los sucesos y motivo de rompimiento de la relación. Las relaciones fueron resignificadas durante la historia de las adolescentes, desde las formas en que las narran. En este sentido, se reafirma la idea de Malpica y Fernández (2009), acerca de cómo la forma en que se organizan las circunstancias influyen sobre las comprensiones que se tienen sobre las situaciones.

Por otra parte, los vínculos cambian a lo largo del ciclo vital a partir de los eventos estresantes que estas adolescentes han tenido que vivir. Carrillo, Maldonado, Saldarriaga, Vega y Díaz (2004) plantean que los vínculos se movilizan a lo largo de la vida y cuando se llega a la "adolescencia" se movilizan hacia pares, generalmente amigos, lo cual también influye para que las relaciones hayan sido resignificadas.

Surge, entonces, la pregunta sobre el papel de la significación en la construcción de los vínculos; pareciera que dependiendo del sentido que se le dio a la relación vincular, esta podría ser distinta, más allá del hecho en sí. En estudios como el de 
Marchant (2007) ya se plantean algunas conclusiones al respecto: “... el vínculo entre una madre y un hijo puede ser entendido como el resultante, principalmente, de un proceso cultural, antes que la mera expresión de instinto de conservación del ser humano" (p. 145).

En cuanto la dimensión temporal, esta es fundamental en el mantenimiento de los vínculos (Marchant, 2007) o no con la familia de origen. Cuando las adolescentes ingresan con menos edad a protección resignifican más fácilmente los vínculos con sus familias de origen.

En cuanto a los vínculos con los hermanos, llama la atención el rol parentalizado de las hermanas mayores y cómo este cambia. Las adolescentes asumieron estos roles antes de ingresar a la Institución. En este rol parentalizado, se evidenciaron varias de las cualidades de los vínculos planteadas por Hernández (2010), en algunos momentos la función de supervivencia fue considerada como vital, pues una de estas adolescentes proveía los alimentos a sus hermanos respaldando la idea de Oliva (2004) sobre la importancia de las figuras subsidiarias. Lo cuestionable es: sí estas adolescentes en su infancia no tuvieron un vínculo de apego seguro con sus madres, o las relaciones con ellas no cumplían con varias de las cualidades de un proceso vincular, cómo hacían para proveer cuidados y protección a sus hermanos. Vale la pena profundizar en cómo las adolescentes pudieron generar estas cualidades hacia sus hermanos menores, cuando no las experimentaron hacia ellas por parte de sus padres. El rol de las hermanas cambió al ingreso a la Institución, que asumió, de manera simbólica, el rol de cuidador. Los vínculos también fueron resignificados, por otros factores, como: separación de grupos de hermanos, adopciones en familias distintas y asunción del rol parental por parte de la Institución. Los vínculos con los hermanos se modificaron al interior del sistema, debido a diferentes situaciones, como adopciones, vivir en diferentes espacios de la Institución, estudiar en distintos colegios, convirtiéndose estos también en inciertos. Aunque es de mencionar que en uno de los casos se presentaron diferentes intentos, después de muchos años, por mantener esos vínculos desde su rol pa- rentalizado, ratificándose la idea de Maldonado y Carrillo (2002) sobre cómo los hermanos mayores son una fuente de seguridad para los hermanos menores; sobre todo cuando estos últimos tienen que explorar un nuevo ambiente.

En cuanto a personas que trabajan en la Institución se encontró que los vínculos que establecen las adolescentes con el equipo psicosocial son de ayuda ante dificultades que se les presentan, relacionadas con su situación en el sistema de protección. Uno de los vínculos con más fuerza se construyó con la educadora de hogar. Como lo plantea el estudio de De Brito y Souza (2011), todas las educadoras de hogar, en este caso son mujeres y se encargan de diferentes tareas frente a las adolescentes. Estas personas comparten la cotidianidad con las adolescentes, los fines de semana y noches, momentos en los que hay más tiempo libre. Estas personas cumplen con ciertas características planteadas por Howes (1999), para el apego en las figuras subsidiarias: cuidado personal y permanencia en el tiempo.

Sobre las características de estos vínculos, Hernández (2010) plantea que los procesos de vinculación implican diferentes dimensiones: la filogenética, epigenética, ontogenética y cultural; en esta relación se evidencian elementos de estas dimensiones, como cuidado parental, pero al mismo tiempo cultural, es decir, la significación de cómo se ha definido que se cuida al otro y quién lo debe hacer; la fortaleza del vínculo la relacionan con el significado de mamá, como sucede con el vínculo enunciado del hogar sustituto. También esta relación vincular se enmarca dentro de las formas de realizar tareas que se desarrollan diariamente en el hogar en la relación: organizar, prepararse para acostarse, levantarse, acciones de higiene en las mañanas, ir a desayunar. Desde allí se comparte el planteamiento de Hernández (2010), que defiende la idea de que lo determinante en el sujeto no son solo las experiencias históricas infantiles, sino también las posteriores.

Se hallaron otras figuras fundamentales, relacionadas con la institucionalización: los padrinos y los hogares sustitutos. Cuando los padrinos son constantes, se comunican, preguntan, proveen 
ciertos elementos para que el desarrollo de las adolescentes sea mejor. Por lo tanto, se evidencia la necesidad de hacer estudios que describan en profundidad estas relaciones y que al mismo tiempo puedan intervenir sobre ellas.

Las relaciones con los hogares sustitutos fueron muy significativas. La pregunta es hasta cuándo deben permanecer los niños en un hogar sustituto; en este caso se generan vínculos significativos y cumplen varias de las cualidades de un proceso de vinculación que fortalece procesos de desarrollo. Así, se reafirma la idea de Cyrulnik (2008), en la que plantea que los niños en situación de abandono buscan otras figuras subsidiarias relacionadas con la institucionalización. Sin embargo, llama la atención que ninguna de las adolescentes hace referencia a los profesores de los colegios como figuras significativas, contradiciendo los hallazgos de Mota y Matos (2010).

Un factor que las adolescentes consideraron importante en el establecimiento de los vínculos es el cuidado: expresaron relaciones más fuertes con personas que las han cuidado en situaciones cotidianas, como las rutinas. Como plantea Berger (2004), es fundamental desarrollar contextos, en este caso en las instituciones, en los que ellas se sientan cómodas, escuchadas y puedan confiar en alguien. En este sentido es muy importante el número de niños asignado a cada cuidador (Fernández-Daza, M. y Fernández-Parra, 2013). Además, como lo plantean Skeels, y Sandvik-Nylund, (2012); es necesario generar procesos de participación de los adolescentes. Así, los adolescentes podrían generar más vínculos con las instituciones donde habitan.

En cuanto a las poblaciones vulneradas, es importante continuar con el estudio de los vínculos subsidiarios y cómo estos pueden fortalecer y lograr resignificaciones de las rupturas iniciales que se pudieran haber dado. Además, se deben fortalecer estudios alrededor de los vínculos que generan los niños, niñas y adolescentes que han estado en medida de restablecimiento de derechos al egresar de instituciones de protección. A partir de los relatos se evidencia lo planteado en los estudios de Barudy y Dantagnan (2005), en los que afirman que en protección se dan cambios en los niños por diferentes razones y estos producen efectos en muchas ocasiones negativos sobre la evolución de los niños y adolescentes. Esto produce a su vez una serie de efectos sobre las vinculaciones y su reasignación, que es necesario investigar.

Finalmente, es necesario continuar con estudios acerca de los vínculos, inclusive más allá de estas poblaciones vulnerables, porque el cuidado de los niños y de las niñas ya no está a cargo de una sola figura sino de muchas: madre, padre, niñera, profesores de jardín.

\section{Referencias}

Armus, M., Duhalde, C., Oliver, M. y Woscoboinik, N. (2012). Desarrollo emocional clave para la primera infancia. Argentina: Unicef.

Amar, J., y Berdugo, M. (2006). Vínculos de apego en niños víctimas de violencia intrafamiliar. Piscología desde el Caribe, 18, 1-22. Recuperado el 20 de marzo de 2013 de http://www. redalyc.org/pdf/213/21301802.pdf

Amar, J., Kotliarenko, M., y Abello, R. (2003). Factores psicosociales asociados con la resiliencia en niños colombianos víctimas de violencia intrafamiliar. Investigación y Desarrollo, 11, 162-197. Recuperado el 25 de enero de 2013 de http://www.redalyc.org/ pdf/268/26811107.pdf

Arango, C. (2003). Los vínculos afectivos y la estructura social; una reflexión sobre la convivencia desde la red de la promoción del buen trato. Investigación y Desarrollo, 11, 70-103. Recuperado el 20 de noviembre de 2010 http://www. redalyc.org/pdf/268/26811104.pdf

Barudy, J., y Dantagnan, M. (2005). Los buenos tratos a la infancia: parentalidad, apego y resiliencia. Barcelona: Gedisa.

Berger, C. (2004). Subjetividad adolescente: tendiendo puentes entre la oferta y demanda de apoyo psicosocial para jóvenes. Revista Psykhe, 13, 143-157. DOI: 10.4067/S071822282004000200011. 
Bowlby, J. (1997). El Apego y la Pérdida. Barcelona: Paidós.

Carrillo, S., Maldonado, C., Saldarriaga, L., Vega, L. y Díaz, S. (2004). Patrones de apego en familias de tres generaciones: abuela, madre adolescente, hijo. Revista Latinoamericana de Psicología, 36, 409-430. Recuperado el 15 de mayo de 2012 de http://www.redalyc.org/ pdf/805/80536304.pdf

Castelblanco C., Moreno J., Moreno L., Sánchez S., Garzón D., y Duque R. (2012). Construcción narrativa de los vínculos de familias sustitutas permanentes del programa aldeas infantiles SOS. Bogotá. Psicogente, 15(28), 428-444. Recuperado el 5 de enero de 2014 de http://connection.ebscohost.com/c/articles/87311588/construcci-n-narrativa-de-losv-nculos-de-familias-sustitutas-permanentesdel-programa-aldeas-infantiles-sos-bogota

Cyrulnik, B. (2008). Bajo el signo del vínculo. Una historia natural del apego. Barcelona: Gedisa.

Dabas, E., y Najmanovich, D. (1995). Redes el lenguaje de los vínculos. Hacia la reconstrucción y el fortalecimiento de la sociedad civil. Buenos Aires: Paidós.

De Brito, C., y Souza, J. (2011). Qualidade de vida dos educadores sociais em abrigos de proteção a crianças e adolescentes. (Portuguese). Psicologia: Teoria E Prática, 13(1), 89-100. Recuperado el 17 de agosto de 2013 de http://connection.ebscohost.com/c/articles/67010690/qualidade-de-vida-dos-educadores-sociais-em-abrigos-de-prote-o-crian-ase-adolescentes

Fernández, J., Malpica M., y Fernández, M. (2009). Codificación y análisis diferencial de los problemas de los adolescentes acogidos en centros de protección. Universitas Psicológica, 9, 841-848. Recuperado el 15 de abril de 2012 de http://www.redalyc.org/ pdf/647/64716836017.pdf

Fernández-Daza, M., y Fernández-Parra, A. (2013). Problemas de comportamiento y competen- cias psicosciales en niños y adolescentes institucionalizados. Universitas Psychologica, 12(3), 1-31. Recuperado el 26 de enero de 2014 de: http://revistas.javeriana.edu.co/index.php/revPsycho/article/view/2582/5802

García-Calvo, P., y García, B. (2000). Secuelas del maltrato en los esquemas de representación y efectos de los estilos de vida en centros de acogida. Psicología Educativa, 6(1), 51-74.

Henao, G., y García, M. (2012). Interacción familiar y desarrollo emocional en niños y niñas. Revista Latinoamericana de Ciencias Sociales, Niñez y Juventud, 7(2), 785-80.

Hernández, A., y Bravo, F. (2004). Vínculos, redes y ecología. Hallazgos, 1, 111-129.

Hernández, A. (2010). Vínculos, individuación y ecología humana: hacia una psicología compleja. Bogotá: Universidad Santo Tomás.

González, L., y Méndez L. (2002). Relación entre autoestima, depresión y apego en adolescentes urbanos en una comuna de Concepción, Chile. Terapia psicológica, 24(1), 5-14. Recuperado el 17 de noviembre de 2012 de http:// www.redalyc.org/pdf/785/78524101.pdf

Howes, C. (1999). Attachment relationships in the context of multiple caregivers. En J. Cassidy y P.R., Shaver (eds.), Handbook of Attachment: Theory, Research, and Clinical Applications. New York: The Guilford Press.

Maldonado, C., y Carrillo, S. (2002). El vínculo de apego entre hermanos: un estudio exploratorio con niños colombianos de estrato bajo. Suma Psicológica, 9, 107-132. Recuperado el 15 de noviembre de 2012 de http://www.redalyc.org/pdf/805/80538105.pdf

Marchant, M. (2007). Reflexiones en torno a los procesos de institucionalización y separación afectiva temprana en el contexto de un hogar de protección de lactantes. Revista de Psicología, 16, 123-146. Recuperado el 16 de mayo de 2013 de http://redalyc.uaemex.mx/redalyc/src/inicio/ArtPdfRed.jsp?iCve=26416105 
Melendro, M. (1998). Adolescentes protegidos. Madrid: Comunidad de Madrid.

Melendro, M. (2013). Estrategias de intervención e investigación. Melendro, M., y Rodríguez, A. (coords.). Intervención con menores y jóvenes en dificultad social, 233-280. Madrid: UNED.

Melendro, M., y Cruz, L. (2013). Los escenarios de la intervención. Melendro, M., y Rodríguez, A. (coords.). Intervención con menores y jóvenes en dificultad social, 85-140. Madrid: UNED.

Moreno-Manso, J., García-Baamonde M., Guerrero-Barona, E., y Blázquez-Alonso M. (2010). Competencia pragmática y adaptación psicosocial en niños sujetos a medidas de protección infantil. Salud Mental, 33, 333-340. Recuperado el 10 de enero de 2014 de http:// bdatos.usantotomas.edu.co:2099/ehost/ pdfviewer/pdfviewer?vid $=16 \&$ sid $=05 f 2 c 885$ ee4c-4f7f-82b6-7ba607e4ea71\% 40sessionmgr 4001\&hid $=4204$
Mota, C., y Matos, P. (2010). Adolescentes institucionalizados: papel das figuras significativas na predição da assertividade, empatia e autocontrolo. Aná. Psicológica, 28(2), 245-254. Recuperado el 10 de enero de 2014 de http:// bdatos.usantotomas.edu.co:2099/ehost/ pdfviewer/pdfviewer?vid $=17 \&$ sid $=05 f 2 c 885$ ee4c-4f7f-82b6-7ba607e4ea71\%40sessionmgr 4001\&hid $=4204$

Skeels, A., y Sandvik-Nylund, M. (2012). La participación de los adolescentes en la protección: beneficios para todos. Revista Migraciones Forzadas, 40, 9-10. Recuperado el 25 de enero de 2014 de http://www.fmreview.org/es/ pdf/RMF40/RMF40\%20Skeels-SandvikNylund. pdf

Oliva, A. (2004). Estado actual de la teoría del apego. Revista de Psiquiatría y Psicología del Niño y del Adolescente, 4, 65-81. Recuperado el 18 de agosto de 2012 de http://chitita.uta. $\mathrm{cl} /$ cursos/2012-1/0000636/recursos/r-9.pdf 